\title{
Optimal Placement of a UAV to Prolong the Lifetime of Indoor Wireless Devices
}

\author{
Hazim Shakhatreh \\ Department of Telecommunications Engineering, Hijjawi Faculty for Engineering Technology, \\ Yarmouk University, Irbid 21163, Jordan
}

Copyright (c) 2019 by authors, all rights reserved. Authors agree that this article remains permanently open access under the terms of the Creative Commons Attribution License 4.0 International License

\begin{abstract}
Unmanned aerial vehicles (UAVs) can be used as aerial wireless base stations when cellular networks go down. Prior studies on UAV-based wireless coverage typically consider outdoor scenarios, where a UAV and wireless devices are outdoor. In this paper, the problem of UAV placement is studied, where the goal is to find the optimum location of a single UAV that prolongs the lifetime of indoor wireless devices. First, a realistic Indoor-Outdoor path loss model is presented and the tradeoff introduced by this model is described. Then, the problem of UAV placement is formulated, where the goal is to find the optimum UAV location that prolongs the lifetime of indoor wireless devices. It can be proven that the constraint sets of the problem can be represented by a convex set in terms of three variables. To this end, an algorithm to find the optimum UAV location is proposed. Simulation results are presented to validate the effectiveness of the proposed algorithm.
\end{abstract}

Keywords Unmanned Aerial Vehicles (UAVs), Indoorto-Outdoor Path Loss Model, Lifetime of Wireless Network, Emergency Response, Convex Optimization

\section{Introduction}

During disaster situations, UAVs can be deployed to provide continuous wireless coverage when the terrestrial network goes down [1]. They can also be used to provide seamless wireless coverage when the cellular network service is not available or it is unable to serve all wireless devices[2].

Prior studies on UAV-based wireless coverage typically consider outdoor scenarios, where a UAV and wireless devices are outdoor. Only a few studies consider the indoor scenarios in which an outdoor UAV is used to provide wireless coverage for indoor wireless devices. The authors in [3] suggest using a single UAV to provide indoor wireless coverage under disas-

\footnotetext{
${ }^{1}$ Part of this work was presented in IEEE IWCMC 2018 [7].
}

ter situations, where the placements of indoor wireless devices are symmetric across the dimensions of each floor, and apply the gradient descent algorithm to find an efficient placement of the UAV that minimizes the transmit power. In [4], the authors assume that the placements of indoor wireless devices are uniformly distributed in each floor and apply the particle swarm optimization algorithm to find an efficient location of a single UAV that minimizes the transmit power required to cover the indoor wireless devices. The authors in [5] minimize the number of UAVs required to provide indoor wireless coverage and prove that this problem is NP-complete. Due to the intractability of the problem, they apply clustering to minimize the number of UAVs required for indoor wireless coverage. In [6], the authors maximize the indoor wireless coverage using UAVs equipped with directional antennas. They propose two methods for the placements of UAVs; providing indoor wireless coverage from one building side and from two building sides. However, the authors of these research studies assume downlink scenarios from UAVs to indoor users.

In post-disaster scenarios such as earthquakes or floods, wireless devices may not be able to connect with remoteundamaged terrestrial cellular network due to the limited transmit power of these devices [7]. Due to physical damage to energy infrastructure, they may also not be able to recharge their batteries as shown in Figure 1. In the case of Hurricane Irma, at the peak of the power blackout following Hurricane Irma, over $36 \%$ of all accounts in Florida were without electricity [9]. In case of an emergency, one of the first things we do is to pick up our mobile phones to learn about the emergency event, locate family and friends, and report their safety [10]. In this paper, the motivation is to explore how the location of a single UAV can prolong the time durations of uplink transmissions of indoor wireless devices when a UAV is used to provide indoor wireless coverage under disaster situations. The authors in [7] and [8] study this problem under the assumption that the users are outdoor. To the best of our knowledge, this is the first work that proposes using a single UAV to prolong the sum of time durations of uplink transmissions under disaster situations, where the indoor users are not able to recharge their wireless devices due to physical damage to energy infrastruc- 


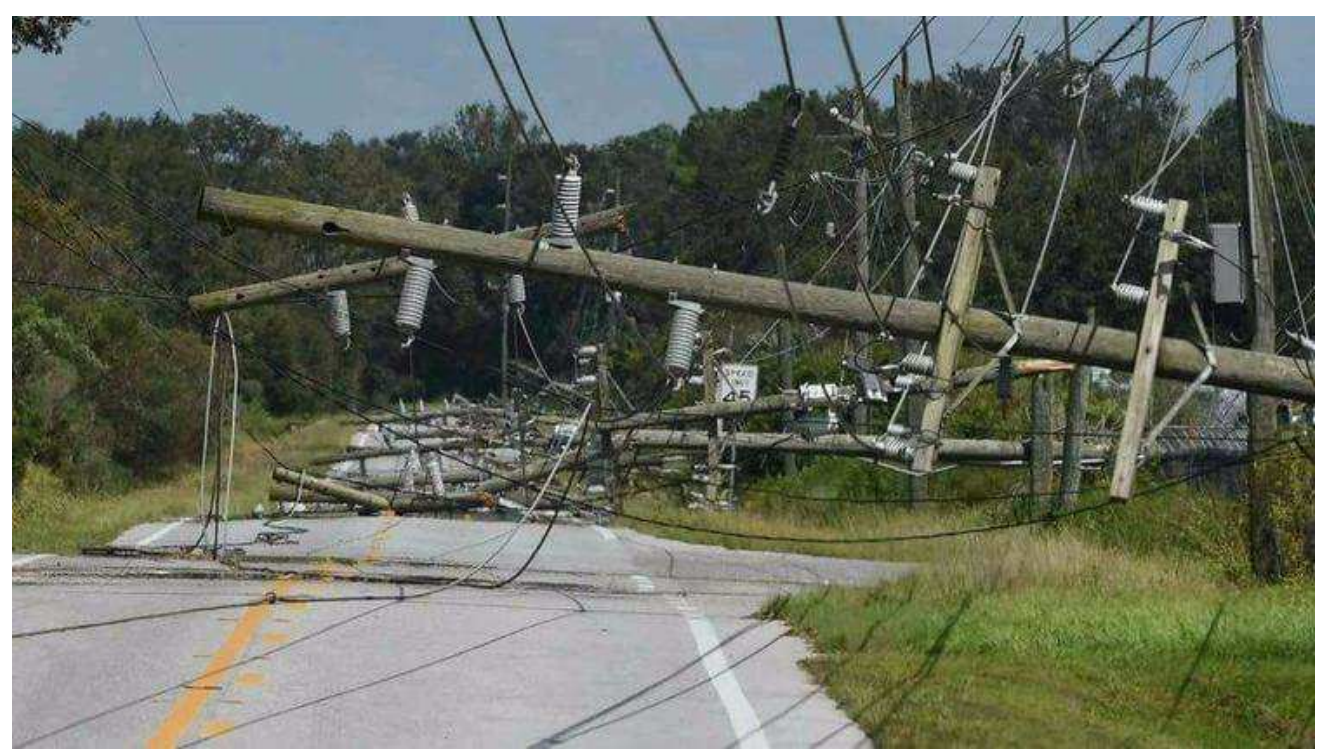

Figure 1. Downed power lines following Hurricane Irma [14].

ture. The main contributions are summarized as follows:

- A realistic Indoor-Outdoor path loss model is presented and the tradeoff introduced by this model is described.

- The problem of UAV placement is formulated, where the goal is to find the optimum UAV location that prolongs the lifetime of indoor wireless devices.

- The constraint sets of the problem are proved that can be represented by a convex set in terms of three variables and an algorithm is proposed to find the optimum location of UAV.

The rest of this paper is organized as follows. In Section 2, the system model is described. In Section 3, the problem of UAV placement is formulated, where the goal is to find the optimum UAV location that prolongs the lifetime of indoor wireless devices. In Section 4, an algorithm is proposed to find the optimum UAV location. Finally, the numerical results are presented in Section 5 and concluding remarks are made in Section 6.

\section{System Model}

\subsection{System Settings}

In Figure 2, $|U|$ indoor wireless devices are assumed to be placed inside a building, and $\left(x_{k}, y_{k}, z_{k}\right)$ is used to represent the placement of indoor wireless device $k \in U$. The dimensions of the building are $X^{b} \times Y^{b} \times Z^{b}$. Also, let $\left(X^{u}, Y^{u}, Z^{u}\right)$ denote the placement of the UAV, let $D_{k}^{u}$ be is the 3D outdoor path-length between the UAV and the external wall, let $\theta_{k}$ be the incidence angle, and let $d_{i n, k}$ be the 2D indoor distance of wireless device $k$ inside the building. Each wireless device $k \in U$ has a residual energy $e_{k}$ with maximum transmit power $P^{\max }$. The indoor wireless devices must be served by the aerial base station to collect the information. An uplink scenario is assumed in which the indoor wireless devices utilize a frequency division multiple access (FDMA) technique to send information to the UAV at a desired data rate $r$. FDMA allocates one subchannel to each wireless device for transmission and hence the channels do not interfere with one another. Also, each indoor device $k \in U$ is considered to be served by the aerial base station for a time $t_{k}$ seconds and this time depends on the residual energy of the wireless device $e_{k}$. The time duration of uplink transmission $t_{k}$ must be greater than or equal to $t_{t h}$. The indoor wireless devices can send the values of residual energies to the UAV using control messages [11].

\subsection{Indoor-Outdoor Path Loss Model}

The Outdoor-Indoor path loss model, certified by the ITU [12], is not appropriate when the uplink scenario for indoor users is considered, because this model assumes that the transmitter is outdoor and the receivers are indoor. In this paper, the Indoor-Outdoor path loss model is adopted [13]. The path loss between indoor user $k$ and the $\mathrm{UAV}$ is given as follows:

$$
\begin{array}{r}
L_{k}^{u}=L_{o}+L_{b}+L_{i}=\left(w_{1}+w_{2} \log _{10}\left(D_{k}^{u}+d_{i n, k}\right)\right) \\
+\left(w_{3}+w_{4}\left(1-\cos \theta_{k}\right)^{2}\right)+\left(w_{5} d_{i n, k}+w_{6} q\right)
\end{array}
$$

where $L_{o}$ is the outdoor propagation loss, $L_{b}$ is the building penetration loss and $L_{i}$ is the indoor loss. Also, $d_{i n, k}$ is the indoor horizontal path-length, $D_{k}^{u}$ is the $3 \mathrm{D}$ outdoor path-length, $\theta_{k}$ is the incidence angle on the external wall, $q$ is the number of apartment or office separations along the indoor horizontal path and $w_{1}-w_{6}$ are constants.

\subsection{Path Loss Model Tradeoff}

There is a key tradeoff in the Indoor-Outdoor path loss model when the horizontal distance between the aerial base station and an indoor wireless device changes. When this horizontal distance increases, the outdoor propagation loss (i.e., $L_{o}$ ) increases as $D_{k}^{u}$ increases, while the building penetration loss 


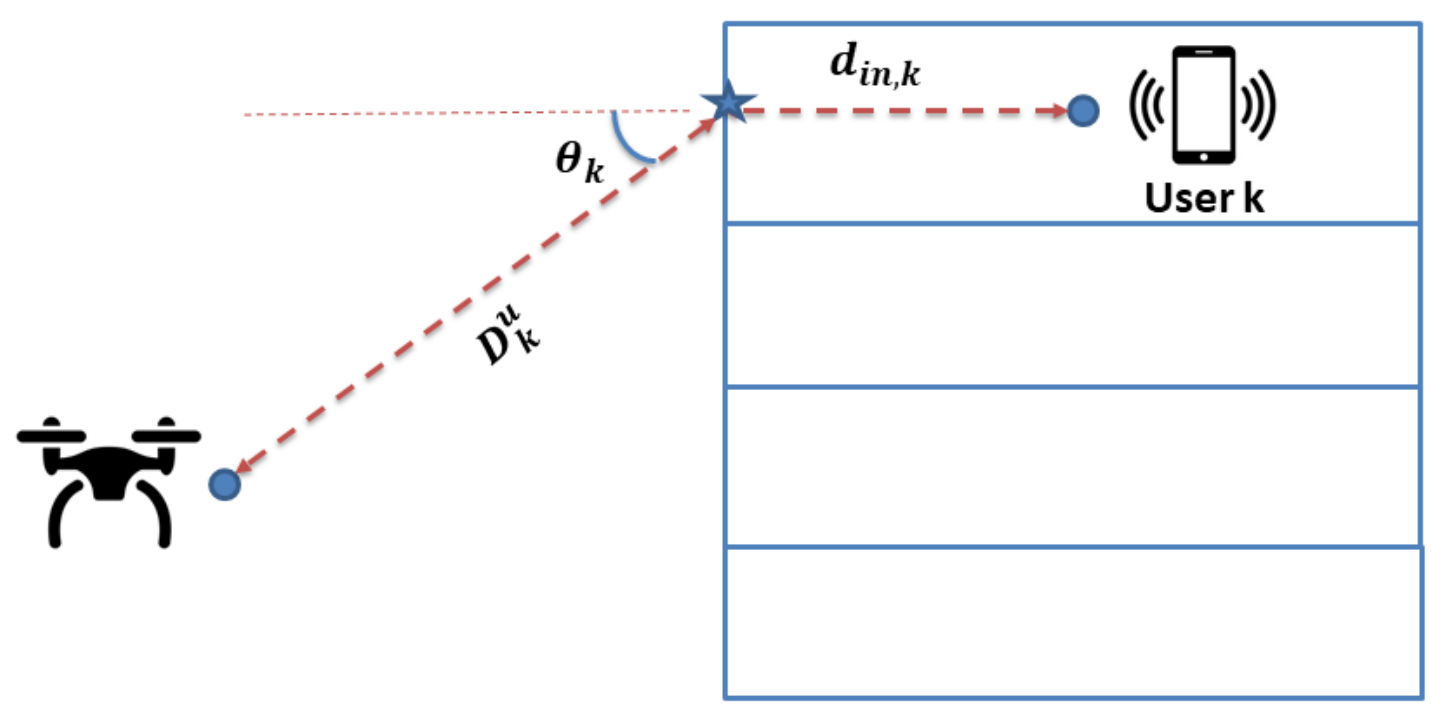

Figure 2. Indoor user transmitting data to a UAV.

(i.e., $L_{b}$ ) decreases as the incident angle (i.e., $\theta_{k}$ ) decreases. Similarly, when this horizontal distance decreases, the outdoor propagation loss (i.e., $L_{o}$ ) decreases as $D_{k}^{u}$ decreases, while the building penetration loss (i.e., $L_{b}$ ) increases as the incident angle (i.e., $\theta_{k}$ ) increases.

Under the assumption that the locations of indoor wireless devices and the location of the UAV are fixed, the path loss between indoor wireless device $k$ and the UAV can be represented as a function of the incidence angle $\theta_{k}$ :

$$
\begin{gathered}
L_{k}^{u}\left(\theta_{k}\right)=\left(w_{1}+w_{2} \log _{10}\left(\frac{\Delta H_{k}^{u}}{\sin \theta_{k}}+d_{i n, k}\right)\right)+\left(w_{3}+\right. \\
\left.w_{4}\left(1-\cos \theta_{k}\right)^{2}\right)+\left(w_{5} d_{i n, k}+w_{6} q\right)
\end{gathered}
$$

where $\Delta H_{k}^{u}$ is the altitude difference between the UAV and the indoor wireless device $k$. In Figure 3, the minimum transmit power of the indoor wireless device required to achieve the threshold Signal-to-noise ratio (SNR) is demonstrated. The minimum transmit power of indoor wireless device is given by:

$$
\begin{gathered}
P_{t x, \min }(d B)=P_{r x, t h}+L_{k}^{u} \\
P_{r x, t h}(d B)=N+\nu
\end{gathered}
$$

Here, $P_{r x, t h}$ is the minimum received power, $N$ is the noise power (equals $-120 \mathrm{dBm}$ ) and $\nu$ is the threshold SNR (equals $37 \mathrm{dBm}$ ). The numerical results show that there is an optimum incidence angle that minimizes the total transmit power of the indoor wireless device $k$. The optimum incident angle $\theta$ that minimizes the transmit power required to satisfy the threshold SNR between the indoor wireless device and the UAV is $48^{\circ}$. Note that this angle is also the optimum angle for the downlink scenario, from the UAV to an indoor wireless device [3].

\section{Problem Formulation}

Consider a transmission between an indoor wireless device located at $\left(x_{k}, y_{k}, z_{k}\right)$ and a UAV located at $\left(X^{u}, Y^{u}, Z^{u}\right)$.
The data rate for indoor wireless device $k$ is given by:

$$
C_{k}=B_{k} \log _{2}\left(1+\frac{p_{k} / L_{k}}{N}\right)
$$

where $B_{k}$ is the transmission bandwidth of indoor wireless device $k, p_{k}$ is the transmit power from indoor wireless device $k$ to the UAV, $L_{k}$ is the path loss between indoor wireless device $k$ and the UAV and $N$ is the noise power.

Let us assume that all indoor wireless devices have the same data rate $r$ and each indoor wireless device has a channel with bandwidth equals $B /|U|$, where $B$ is the UAV bandwidth and $|U|$ is the number of indoor wireless devices. Then, the minimum power required to satisfy this data rate for each indoor wireless device is given by:

$$
p_{k}=\left(2^{\frac{r \cdot|U|}{B}}-1\right) \cdot N \cdot L_{k}
$$

Our goal is to find the optimum placement of the UAV such that the lifetime of indoor wireless devices defined by $S$ is prolonged. Here, the lifetime $S$ represents the sum of time durations of uplink transmissions of indoor wireless devices. Our problem can be formulated as:

$$
\begin{array}{cc}
\max _{\left(X^{u}, Y^{u}, Z^{u}\right), t_{k}} S=\sum_{k=1}^{|U|} t_{k}=\sum_{k=1}^{|U|} \frac{e_{k}}{p_{k}} & \\
\text { subject to } & \\
p_{k} \leq P^{\max } & \forall k \in U \\
t_{k} \geq t_{t h} & \forall k \in U \\
t_{k} \cdot p_{k} \leq e_{k} & \forall k \in U \\
X^{u} \in\left[x_{\min }, x_{\max }\right] & \\
Y^{u} \in\left[y_{\min }, y_{\max }\right] & \\
Z^{u} \in\left[z_{\min }, z_{\max }\right] &
\end{array}
$$

Here, constraint set (7.a) guarantees that the transmit power of each indoor wireless device is not exceed its maximum transmit power $P^{\max }$. Constraint set (7.b) ensures that each indoor 
wireless device $k \in U$ is served by aerial base station for a time greater than $t_{t h}$ seconds. Constraint set (7.c) guarantees that the total energy consumed by indoor wireless device is not exceed its residual energy $e_{k}$. Constraints (7.d-7.f) show the maximum and minimum allowed values for $X^{u}, Y^{u}$ and $Z^{u}$. Despite the optimization problem has a number of nonlinear constraints, it can be solved by proving that the constraint sets of the problem can be represented by the intersection of convex sets.

Theorem 1 Under the assumption that the altitude of a UAV is fixed, the constraint sets in optimization problem (7) can be represented by the intersection of convex sets and the region formed by this intersection is also a convex set.

Proof 1 Let us assume that the altitude of a UAV $Z^{u}$ is fixed. From (2) and (6), the transmit power of indoor wireless device $k$ is given by:

$$
p_{k}=\left(2^{\frac{r \cdot|U|}{B}}-1\right) \cdot N \cdot L_{k}\left(\theta_{k}\right)=Q \cdot L_{k}\left(\theta_{k}\right)
$$

where $Q$ is a constant and equals $\left(2^{\frac{R \cdot|I|}{B}}-1\right) . N$. Now, to satisfy constraint set (7.a), $p_{k}$ must be less than $P^{\text {max }}$. From (8), the range of incidence angles $\theta_{1}$ that satisfies the constraint set (7.a) is given by:

$$
L_{k}\left(\theta_{k}\right) \leq \frac{P^{\max }}{Q}
$$

The range of incidence angles $\theta_{1}$ (less than $90^{\circ}$ ) represents a convex set. To satisfy constraint sets (7.b) and (7.c), $p_{k}$ must be less than $\frac{e_{k}}{t_{t h}}$. From (8), the range of incidence angles $\theta_{2}$ that satisfies constraint sets (7.b) and (7.c) is given by:

$$
L_{k}\left(\theta_{k}\right) \leq \frac{e_{k}}{t_{t h} \cdot Q}
$$

The range of incidence angles $\theta_{2}$ also represents a convex set. The range of distances that satisfies constraint sets (7.d) and (7.e) is given by a $2 D$ plane. The constraint sets in optimization problem (7) can be represented by the intersection of convex sets and the region formed by this intersection is a convex set [15].

From Theorem 1, the constraints of the optimization problem (7) can be represented as a convex set, when the altitude of a UAV is fixed. In the next theorem, the objective function is proved that it is concave when the altitude of a UAV is fixed. This theorem enables us to find the optimum location for UAV.

Theorem 2 The objective function of (7) is concave when the altitude of a UAV is fixed.

Proof 2 The nonnegative weighted sums preserve the concavity of function [15]. Since $e_{k}>0, \forall k \in U$, we need to prove that (11) is a concave function.

$$
F=\frac{1}{L_{k}\left(\theta_{k}\right)}, \forall k \in U
$$

From Figure 3, we can notice that $L_{k}\left(\theta_{k}\right)$ is a convex function and hence (11) is a concave function.

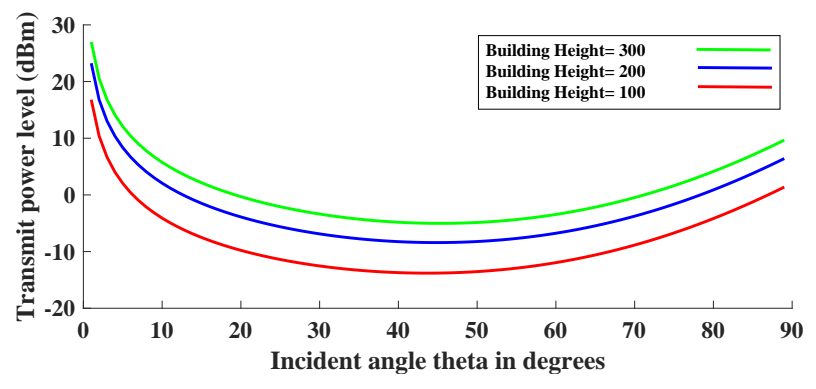

Figure 3. Minimum transmit power of indoor wireless device.

Theorem 2 enables us to find the optimum 2D location for the $\operatorname{UAV}\left(X^{u}, Y^{u}\right)$, when the altitude of UAV $Z^{u}$ is fixed. In order to the optimum 3D location of a UAV, the optimum UAV placement algorithm is proposed in the next section.

\section{Optimum UAV Placement Algorithm}

The proposed algorithm to find the optimum location for a UAV is shown in Algorithm 1 as follows: The inputs are the locations of indoor wireless devices, the maximum transmit power, the residual energy of each indoor wireless device, the data rate, the total bandwidth, the noise power and the threshold time duration of uplink transmission. In steps (9-10), the convex sets $V_{k}$ at different altitudes of a UAV are found. In steps (11-17), the gradient projection algorithm is used to find the optimum 2D location of a UAV $\left(X^{u}, Y^{u}\right)_{\text {opt }}$ at each altitude $z_{k}$ where $n$ is the iteration number, $\gamma$ is a positive step size, $\nabla F$ is the gradient of the objective function in (7) and []$^{+}$denotes the orthogonal projection of a vector onto a convex set. In steps (18-19), the optimum 3D location of a UAV that prolongs the lifetime of indoor wireless devices is found.

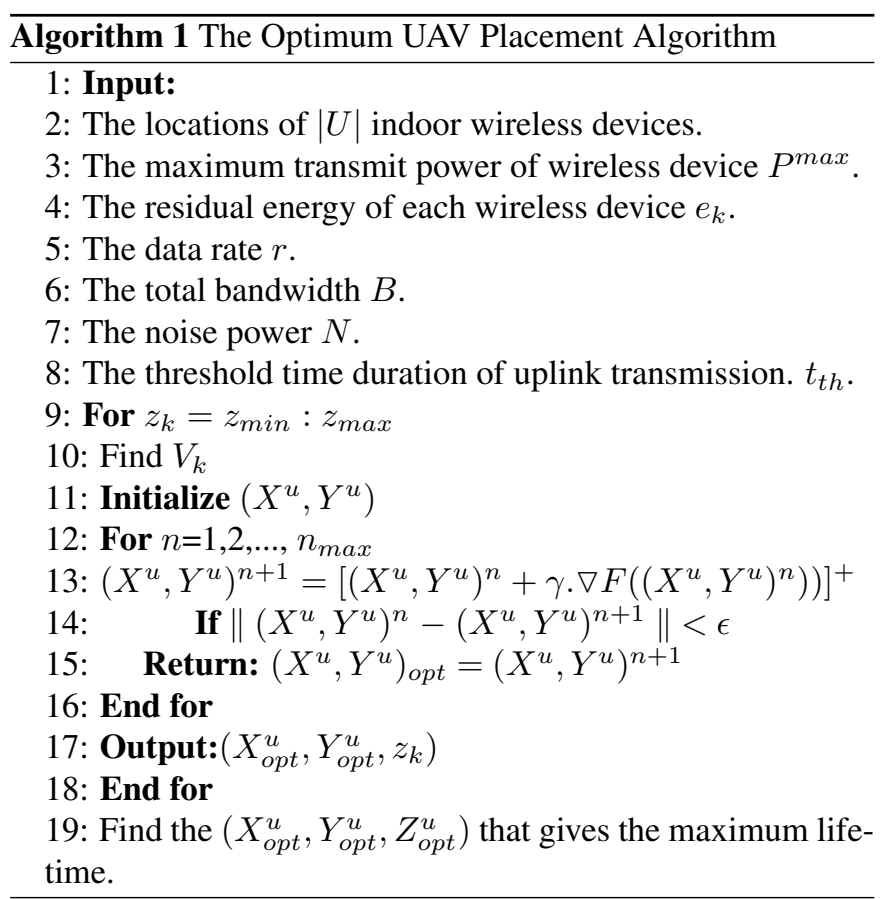




\section{Numerical Results}

In Figure 4.a, 400 indoor wireless devices (blue dots) are uniformly distributed in a building with dimensions $20 \mathrm{~m} \times$ $50 \mathrm{~m} \times 100 \mathrm{~m}$, then Algorithm 1 is used to find the optimum location of the UAV that prolongs the sum of time durations of uplink transmissions. The optimum location for the UAV is $(30,25,57)$ and the optimum lifetime for all indoor devices is 316130 seconds. The average lifetime for each indoor wireless device will be 13.17 minutes. In Figure 4.b, the convergence speed of the Algorithm 1 is showed when the indoor wireless devices are uniformly distributed. The projection of the optimum point is located near the center of the building. This is because the indoor wireless devices that have the highest path losses are placed at the corners of the building and placing the projection of UAV near the center prolongs the total lifetime. Table I lists the parameters used in the numerical analysis.

In Figure 4.c, 400 indoor wireless devices are non-uniformly distributed in the building. To find the optimum location of the UAV that prolongs the sum of time durations of uplink transmissions, Algorithm 1 is used. The optimum location for the UAV is $(30,32,66)$ and the optimum lifetime for all indoor devices is 421570 seconds. The average lifetime for each indoor wireless device will be 17.56 minutes. In Figure 4.d, the convergence speed of the Algorithm 1 is showed when the indoor wireless devices are non-uniformly distributed. The UAV location is near the high density region.

In Figure 4.e, the definition of the lifetime of wireless devices is changed. The new definition of the lifetime represents the time duration of uplink transmission until the first wireless device runs out of energy (Max-Min). Under the disaster situation, it is necessary that we keep all of the indoor wireless devices up as much as we can due to physical damage to energy infrastructure. Therefore, instead of prolonging the lifetime of all indoor wireless devices, the lifetime as defined above is prolonged. The optimum location for the UAV is $(52,24,44)$ and the optimum lifetime is 219 seconds, when the indoor wireless devices are uniformly distributed in the building. As can be seen from the figures, the optimum horizontal point $X^{u}$ increases. This is to recompense the increased building penetration loss due to an increased incident angle. In Figure 4.f, the convergence speed of the algorithm is showed when the indoor wireless devices are uniformly distributed.

In Figure 4.g, the indoor wireless devices are non-uniformly distributed in the building. The objective is finding the optimum location of the UAV that prolongs the time duration of uplink transmission until the first wireless device runs out of energy (Max-Min). The optimum location for the UAV is $(57,34,61)$ and the optimum lifetime is 300 seconds. In Figure 4.h, the convergence speed of the algorithm is showed when the indoor wireless devices are non-uniformly distributed.

\section{Conclusions}

In this paper, the problem of UAV placement is studied, where the goal is to find the optimum location of a single UAV that prolongs the lifetime of indoor wireless devices. First, a realistic Indoor-outdoor path loss model is presented and the tradeoff introduced by this model is described. Then, the problem of UAV placement is formulated, where the goal is to find the optimum UAV location that prolongs the lifetime of indoor wireless devices. The constraint sets of problem are proved that can be represented by a convex set in terms of three variables and an algorithm is proposed to find the optimum location of UAV. The analysis is validated by simulations and the effectiveness of the proposed algorithm is demonstrated under different cases. In order to model more realistic scenarios, different types of user distribution can be considered as future work. Also, the problem of prolonging the lifetime of wireless devices can be studied using multiple UAVs.

\section{REFERENCES}

[1] H. Shakhatreh, A. Khreishah, J. Chakareski, H. Bany Salameh, and I. Khalil. On the continuous coverage problem for a swarm of UAVs. 2016 IEEE 37th Sarnoff Symposium, 130-135, 2016.

[2] H. Shakhatreh, A. Sawalmeh, A. Al-Fuqaha, Z. Dou, , E. Almaita, I. Khalil, N. Othman, A. Khreishah, and M. Guizani. Unmanned Aerial Vehicles: A Survey on Civil Applications and Key Research Challenges. IEEE Access, 48572-48634, 2019.

[3] H. Shakhatreh, A. Khreishah, and B. Ji. Providing wireless coverage to high-rise buildings using UAVs. 2017 IEEE International Conference on Communications (ICC), 1-6, 2017.

[4] H. Shakhatreh, A. Khreishah, A. Alsarhan, I. Khalil, A. Sawalmeh, and N. Othman. Efficient 3D placement of a UAV using particle swarm optimization. 2017 8th International Conference on Information and Communication Systems (ICICS), 258-263, 2017.

[5] H. Shakhatreh, A. Khreishah, and I. Khalil. Indoor Mobile Coverage Problem Using UAVs. IEEE Systems Journal, 1-12, 2018.

[6] H. Shakhatreh, A. Khreishah, N. Othman, and A. Sawalmeh. Maximizing indoor wireless coverage using UAVs equipped with directional antennas. 2017 IEEE 13th Malaysia International Conference on Communications (MICC), 175-180, 2017.

[7] H. Shakhatreh and A. Khreishah. Optimal Placement of a UAV to Maximize the Lifetime of Wireless Devices. 2018 14th International Wireless Communications \& Mobile Computing Conference (IWCMC), 1225-1230, 2018.

[8] H. Shakhatreh, A. Khreishah, and B. Ji. UAVs to the Rescue: Prolonging the Lifetime of Wireless Devices Under Disaster Situations. Submitted to IEEE Transactions on Green Communications and Networking, 2019.

[9] D. Mitsova, A. Esnard, A. Sapat, and B. Lai. Socioeconomic vulnerability and electric power restoration timelines in Florida: the case of Hurricane Irma. Natural Hazards, 689-709, 2018.

[10] M. Deruyck, J. Wyckmans, W. Joseph, and L. Martens. Designing UAV-aided emergency networks for large-scale disaster scenarios. EURASIP Journal on Wireless Communications and Networking, 79, 2018. 
Table 1. Parameters in numerical analysis

\begin{tabular}{|c|c|}
\hline Dimensions of the building & $20 \mathrm{~m} \times 50 \mathrm{~m} \times 100 \mathrm{~m}$ \\
\hline Number of wireless devices $U$ & 400 devices \\
\hline Maximum number of iterations $n_{\max }$ & 100 \\
\hline Maximum transmit power of wireless device $P^{\max }$ & $0.5 \mathrm{watt}$ \\
\hline Energy of each wireless device $e_{k}$ in joule & $4500+13500^{*}$ rand $(200,1)$ \\
\hline Data rate $r$ & $1 \mathrm{Mbps}$ \\
\hline Total bandwidth $B$ & $50 \mathrm{MHz}$ \\
\hline The noise power $N$ & $1 \times 10^{-14}$ \\
\hline The carrier frequency & $2 \mathrm{Ghz}$ \\
\hline Threshold time duration of uplink transmission $t_{t h}$ & $200 \mathrm{~seconds}$ \\
\hline$\left[x_{\min }, x_{\max }\right]$ & {$[25,100]$} \\
\hline$\left[y_{\min }, y_{\max }\right]$ & {$[0,50]$} \\
\hline$\left[z_{\min }, z_{\max }\right]$ & {$[10,100]$} \\
\hline
\end{tabular}

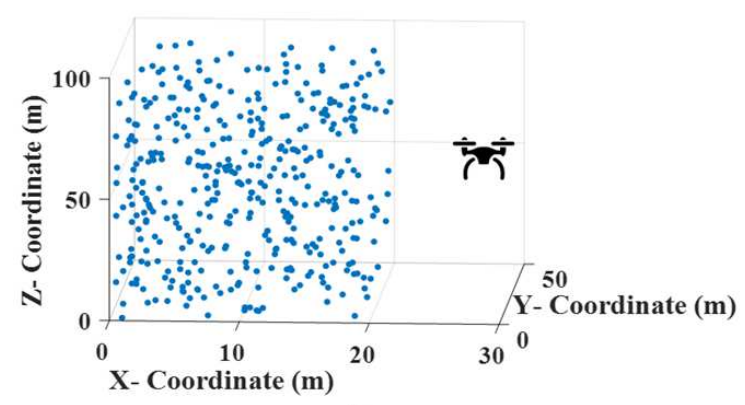

(a)

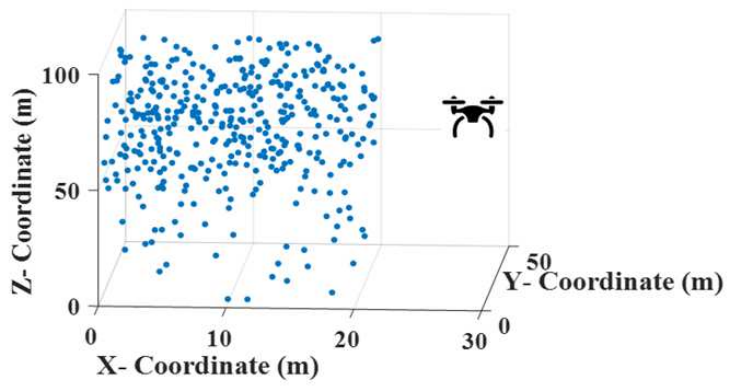

(c)

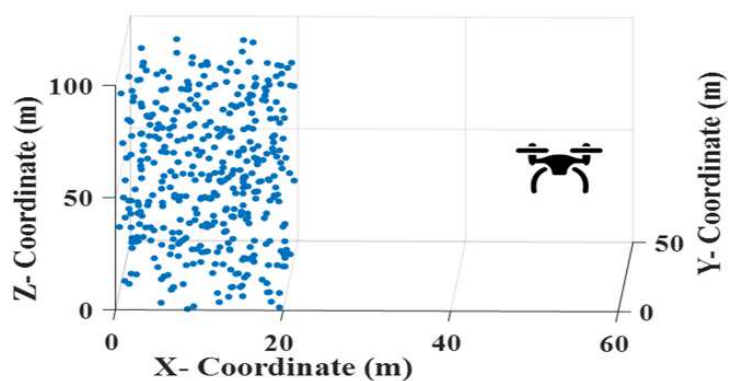

(e)

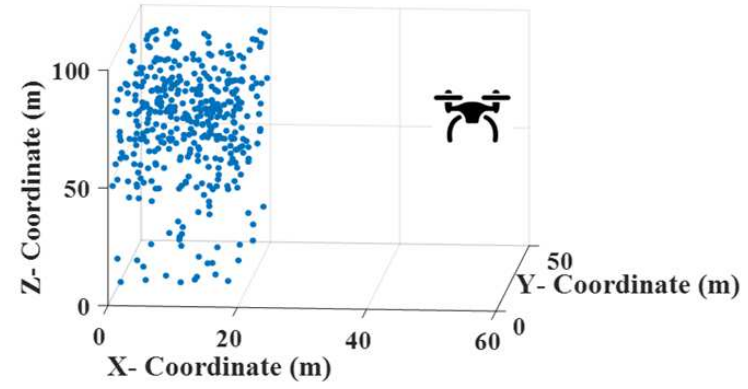

(g)

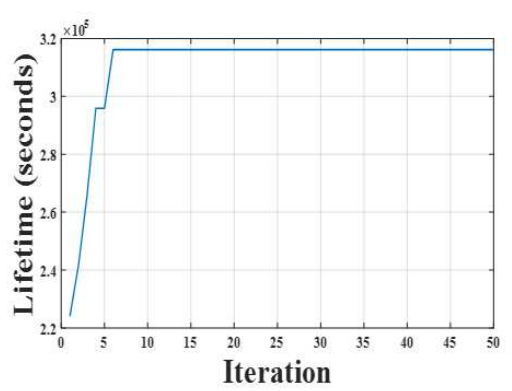

(b)

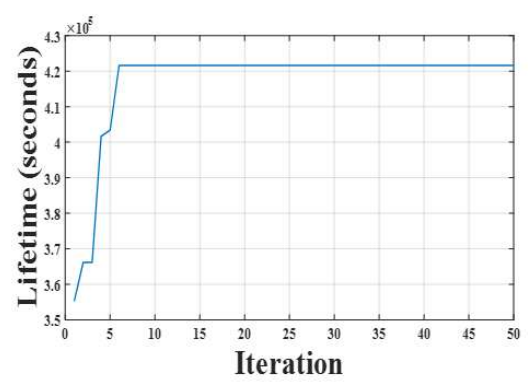

(d)

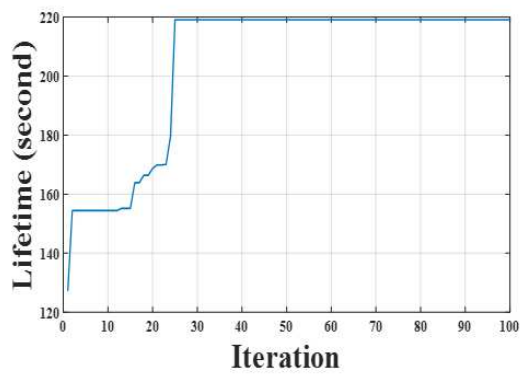

(f)

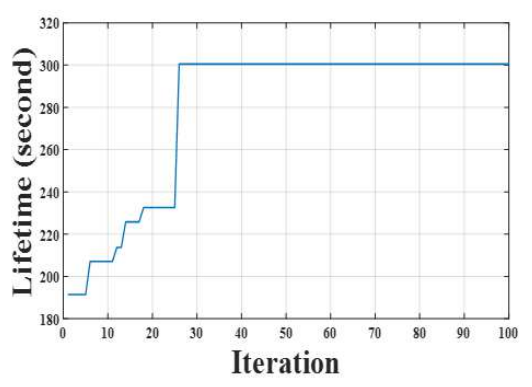

(h)

Figure 4. The optimum 3D placement of the UAV and the convergence speed of the algorithm. 
[11] L. Gupta, R. Jain, and G. Vaszkun. Survey of important issues in UAV communication networks. IEEE Communications Surveys \& Tutorials, 1123-1152, 2016.

[12] M.Series. Guidelines for evaluation of radio interface technologies for IMT-Advanced. Report ITU, 2009.

[13] Y. Corre, J. Stephan, and Y. Lostanlen. Indoor-to-outdoor pathloss models for femtocell predictions. 2011 IEEE 22nd International Symposium on Personal Indoor and Mobile Radio Communications (PIMRC), 824-828, 2011.

[14] M. Comas. Hurricane Irma: State legislators grill Duke Energy about power outages. [Online]. Available: https://www.orlandosentinel.com (Accessed on May 2019).

[15] S. Boyd and L. Vandenberghe. Convex optimization. Cambridge university press, 2004. 